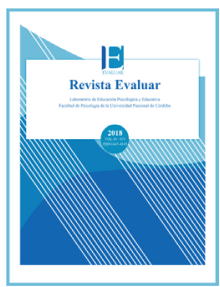

\title{
Propiedades psicométricas de la Escala de Victimización Escolar entre Iguales (VE-I)
}

\section{Psychometric Properties of the Peer School Victimization Scale (VE-I)}

\author{
María-Jesús Cava ${ }^{*}$, Sofía Buelga ${ }^{1}$ \\ 1 - Universidad de Valencia (España)
}

\author{
Introducción \\ Método \\ Resultados \\ Discusión \\ Referencias
}

Recibido: 22/11/2017 Revisado: 26/12/2017 Aceptado: 30/12/2017

\section{Resumen}

La violencia escolar entre iguales tiene graves consecuencias para las víctimas. La victimización puede ser directa, mediante agresiones físicas y verbales, y relacional mediante exclusión y rechazo social. El objetivo de este estudio fue analizar las propiedades psicométricas de la escala de Victimización Escolar entre Iguales (VE-I) que consta de 11 ítems relativos a diferentes formas de victimización. En este estudio participaron 1389 adolescentes $(50.45 \%$ varones), de entre 11 y 15 años $(\mathrm{M}=13.37, \mathrm{DE}=1.23)$. La estructura de la escala fue analizada mediante análisis factorial exploratorio y análisis factorial confirmatorio, por lo que se utilizaron dos submuestras diferentes. Los resultados constatan la existencia de tres factores (victimización fisica, victimización verbal y victimización relacional), con coeficientes de confiabilidad superiores a $\alpha=.70$ y correlaciones negativas con autoconcepto social y satisfacción con la vida. La escala analizada tiene adecuadas propiedades psicométricas y puede ser útil en investigaciones e intervenciones sobre esta temática.

Palabras clave: victimización escolar, victimización entre iguales, adolescencia, escala, propiedades psicométricas

\begin{abstract}
School violence among peers has serious consequences for the victims. Victimization can be direct, through physical and verbal aggression, and relational, through exclusion and social rejection. The objective of this study was to analyze the psychometric properties of the Peer School Victimization Scale (VE-I), which consists of 11 items related to different forms of victimization. 1389 adolescents ( $50.45 \%$ boys) ranging from 11 to 15 years old $(\mathrm{M}=13.37$, $\mathrm{SD}=1.23)$ participated in this study. The structure of the scale was analyzed by means of exploratory factor analysis and confirmatory factor analysis using two different subsamples. The results show the existence of three factors (physical victimization, verbal victimization and relational victimization), with reliability coefficients higher than $\alpha=$ .70 and negative correlations with social self-concept and life satisfaction. The analyzed scale has adequate psychometric properties and can be useful in research and interventions about this topic.
\end{abstract}

Key words: school victimization, peer victimization, adolescence, scale, psychometric properties

Cómo citar este artículo: Cava, M. J., \& Buelga, S. (2018). Propiedades psicométricas de la Escala de Victimización Escolar entre Iguales (VE-I). Revista Evaluar, 18(1), 40-53. Recuperado de https://revistas.unc.edu.ar/index.php/revaluar

* Correspondencia a: María-Jesús Cava. Dirección Postal: Departamento de Psicología Social, Facultad de Psicología, Universidad de Valencia, Avda. Blasco Ibáñez, 21. CP 46010, Valencia (España). Teléfono: 3496398 34 92, Fax: 349638646 68. e-mail: maria.j.cava@uv.es 


\section{Introducción}

En las últimas décadas se han incrementado considerablemente las investigaciones sobre victimización entre iguales en el contexto escolar (Cava, Buelga, Musitu, \& Murgui, 2010; Kljakovic \& Hunt, 2016; Pouwels, Souren, Lansu, \& Cillessen, 2016). Así, diversos estudios han constatado que un elevado número de niños y adolescentes sufren en diferentes países la violencia de sus compañeros (Bradshaw, Crous, Rees, \& Turner, 2017; Del Barrio et al., 2008; Del Rey \& Ortega, 2008; Estévez, Jiménez, \& Cava, 2016). La victimización entre iguales se define como cualquier acto de agresión ejercido por un niño o adolescente hacia otro de similar edad; la mayoría de estas agresiones se produce en el contexto escolar (Wu, Zhang, Su, \& Hu, 2015). Esta victimización puede manifestarse de forma directa a través de agresiones verbales (gritos, burlas e insultos) y físicas (golpes y empujones) y también de forma indirecta o relacional utilizando medios más sutiles como son la exclusión social del grupo, la difusión de rumores y las amenazas de terminar la amistad (Mehari \& Farrell, 2015).

La victimización de los iguales afecta muy negativamente a la salud de las víctimas, quienes manifiestan con frecuencia síntomas depresivos, ansiedad, sentimientos de soledad, baja autoestima, quejas somáticas, imagen negativa de sí mismos y baja satisfacción con la vida (Guhn, Schonert-Reichl, Gaderman, Hymel, \& Hertzman, 2013; Kerr, Valois, Huebner, \& Drane, 2011; Mehari \& Farrell, 2015; Ostrov \& Kamper, 2015; Reijntjes, Kamphuis, Prinzie, \& Telch, 2010; Wu et al., 2015). Estas consecuencias negativas se producen no sólo como consecuencia de sufrir agresiones directas, físicas o verbales, sino también debido a las agresiones indirectas, las cuales tienen consecuencias igual de graves para el bienestar psicosocial de los niños y adolescentes que las sufren (Card \& Hodges, 2008; Cava, Musitu, Buelga, \& Murgui, 2010; Troop-Gordon, 2017). No obstante, y a pesar de la importancia de las agresiones indirectas, las investigaciones en este ámbito se han centrado mayoritariamente en las consecuencias negativas de la victimización directa y solo recientemente se ha prestado mayor atención a la victimización indirecta o relacional basada en la exclusión social, el rechazo y la difusión de rumores maliciosos (Wu et al., 2015). La victimización indirecta o relacional afecta especialmente a la imagen personal y a la autoconfianza de la víctima y ha sido vinculada en mayor medida con problemas de tipo internalizado tales como ansiedad, depresión y autoconcepto negativo (Wu et al., 2015).

Puesto que todas las formas de victimización entre iguales (física, verbal y relacional) tienen serias consecuencias negativas para la víctima, todas ellas deberían incluirse en los instrumentos de medición. Así, aunque los primeros instrumentos se centraban solo en la medición de la victimización física y verbal, posteriores instrumentos han incorporado también la medición de las formas indirectas de victimización. En esta línea se sitúa, por ejemplo, la escala multidimensional de victimización entre iguales de Mynard y Joseph (2000), la cual permite evaluar tanto la victimización directa como la indirecta.

En lengua española, Cava, Musitu y Murgui (2007), tomando como referencia tanto la escala de Mynard y Joseph (2000) como el cuestionario de experiencias sociales de Crick y Grotpeter (1996), elaboraron una escala de victimización entre iguales en el contexto escolar en la que incluyeron 10 ítems relativos a victimización directa y 10 ítems relativos a victimización indirecta. Los análisis factoriales de esta escala mostraron tres factores: victimización relacional (10 ítems), victimización física (4 ítems) y victimización verbal (6 ítems). La escala de Cava et al. (2007) ha 
sido utilizada en diversos estudios, mostrando por un lado correlaciones positivas con medidas de ansiedad, soledad, sintomatología depresiva, estrés percibido y comunicación familiar negativa $y$, por otro, correlaciones negativas con satisfacción con la vida y con las diferentes dimensiones del autoconcepto, en especial con el autoconcepto social (Buelga, Cava, \& Musitu, 2012; Cava, 2011; Estévez, Jiménez, \& Moreno, 2010; Jiménez, Musitu, Ramos, \& Murgui, 2009).

Sin embargo, aunque esta escala aporta adecuados niveles de confiabilidad y resulta útil para investigaciones en este ámbito, sería conveniente disponer de instrumentos más breves con un número menor de ítems. Una escala más breve puede facilitar la aplicación conjunta de una batería más amplia de instrumentos $\mathrm{y}$, además, puede ser especialmente útil para evaluar la efectividad de programas de intervención diseñados para la prevención e intervención en esta problemática. Con el objetivo de disponer de una escala más breve y con la que igualmente se puedan evaluar las diferentes formas de victimización, se desarrolló la escala de Victimización Escolar entre Iguales (VE-I). Esta escala está basada en la escala de Cava et al. (2007), pero incluye un menor número de ítems respecto de la escala original. En la elaboración inicial de la escala VE-I se incluyeron 12 ítems, adaptados de la escala de Cava et al. (2007), que describen diferentes situaciones de victimización escolar entre iguales de tipo relacional, físico y verbal.

El presente estudio se planteó con la finalidad de analizar las propiedades psicométricas de la escala VE-I. Para analizar su estructura, se llevaron a cabo análisis factoriales exploratorios $\mathrm{y}$ análisis factoriales confirmatorios por medio de dos submuestras. También se analizó la consistencia interna de la escala y su validez convergente mediante el análisis de sus relaciones con dos variables con las que se encuentra teóricamente relacionada: el autoconcepto social y la satisfacción con la vida. Mediante este estudio se pretende aportar un instrumento breve y confiable para la adecuada medición de la victimización entre iguales en el contexto escolar.

\section{Método \\ Participantes}

En esta investigación participaron 1389 adolescentes (50.45\% varones y $49.55 \%$ mujeres) que se encontraban en el último curso de educación primaria obligatoria ( $6^{\circ}$ grado) y en educación secundaria obligatoria en cuatro centros educativos de la provincia de Valencia (España). Los adolescentes participantes tenían entre 11 y 15 años $(\mathrm{M}=13.37, \mathrm{DE}=1.23)$ y su nivel socioeconómico era medio. Para la realización de los análisis factoriales, esta muestra inicial se dividió en dos submuestras. Para ello, se le asignó a cada una de estas submuestras dos de los cuatro centros educativos participantes en la investigación. Así, la primera submuestra estuvo compuesta por 735 adolescentes $(50.4 \%$ chicos $)$ de entre 11 y 15 años $(\mathrm{M}=13.53, \mathrm{DE}=1.11)$ que eran estudiantes de dos centros educativos de la provincia de Valencia (España) y la segunda submuestra por 654 adolescentes (50.5\% chicos) de entre 11 y 15 años $(\mathrm{M}=13.20, \mathrm{DE}=1.35)$ que eran estudiantes de los mismos niveles educativos en otros dos centros educativos de la misma provincia con similares características en cuanto a tamaño de los centros y nivel socioeconómico de las familias.

\section{Instrumentos}

La escala de Victimización Escolar entre Iguales (VE-I) supone una adaptación de la escala de Victimización Escolar de Cava et al. (2007). 
La escala VE-I incluye, al igual que la escala de Cava et al. (2007), ítems relativos a las diferentes formas de victimización entre iguales, pero consta de un número menor de ítems, lo que puede ser de especial utilidad para la administración de esta escala en entornos educativos, tanto con fines de investigación como para la evaluación de la efectividad de programas de intervención. La escala de Victimización Escolar entre iguales (VEI) está compuesta inicialmente por 12 ítems que describen situaciones sufridas por los estudiantes en contextos educativos relacionadas con victimización física, con empujones, golpes o patadas (p. ej., Algún compañero/a me ha pegado o golpea$d o$ ), victimización verbal, mediante gritos, burlas o insultos (p. ej., Algún compañero/a me ha insultado) y victimización relacional, mediante exclusión del grupo, difusión de rumores maliciosos o aislamiento social (p. ej., Algún compañero/a le ha dicho a los demás que no vayan conmigo). Los estudiantes responden a estos ítems mediante una escala tipo Likert con cinco opciones de respuesta $(1=$ Nunca me ha pasado; 2 = Solo me pasó una vez; 3 = Me ha pasado alguna vez en el último mes; 4 = Me ha pasado bastantes veces durante el último mes; 5 = Me pasa muy a menudo).

Para analizar su validez convergente, se utilizaron también otros dos instrumentos: la Subescala de Autoconcepto Social del AF-5 (García \& Musitu, 1999) y la Escala de Satisfacción con la Vida de Diener, Emmons, Larsen y Griffin (1985); adaptada al español por Atienza, Pons, Balaguer y García-Merita (2000). La Subescala de Autoconcepto Social está compuesta por 6 ítems que miden aspectos relativos a la percepción que el adolescente tiene de sí mismo en el ámbito de las relaciones sociales con amigos y compañeros ( $\mathrm{p}$. ej., Tengo muchos amigos/as), y es una de las cinco subescalas que se integran en la escala de Autoconcepto AF-5 (García \& Musitu, 1999). Los adolescentes responden a estos ítems mediante una escala Likert de cinco puntos $(1=$ Nunca; 2 = Pocas veces; 3 = Algunas veces; $4=$ Muchas veces; 5 = Siempre). Su consistencia interna (alfa de Cronbach) en el presente estudio fue de .72 en la primera submuestra y de .78 en la segunda submuestra. La Escala de Satisfacción con la Vida (Diener et al., 1985) evalúa con 5 ítems la satisfacción vital de los adolescentes y aporta un índice general de dicha satisfacción en términos de bienestar subjetivo (p. ej., Mi vida es en la mayoría de los aspectos como me gustaría que fuera). Las opciones de respuesta a estos ítems son cinco ( 1 = Muy en desacuerdo; $2=$ En desacuerdo; $3=$ Ni de acuerdo ni en desacuerdo; $4=D e$ acuerdo; 5 = Muy de acuerdo). Su consistencia interna (alfa de Cronbach) en el presente estudio fue de .70 en la primera submuestra y de .75 en la segunda submuestra.

\section{Procedimiento}

En primer lugar, se mantuvo una reunión con los equipos directivos de los cuatro centros educativos para explicarles los objetivos de esta investigación y solicitar su colaboración. Además, se les ofreció información sobre las dificultades de convivencia en las aulas y asesoramiento sobre posibles intervenciones que, una vez concluido el proceso de recolección de datos, podrían realizarse para prevenir la violencia escolar. Los cuatro centros educativos con los que se hizo contacto aceptaron participar en este estudio. Se envió también una carta informativa a las familias explicándoles los principales objetivos del estudio y solicitando su consentimiento para la participación de sus hijos. Tras la obtención de todos los permisos correspondientes se realizó la aplicación de los instrumentos. Esta aplicación fue llevada a cabo por investigadores expertos en las aulas habituales. Se informó también a los 
adolescentes, previamente a la cumplimentación de los instrumentos, que su participación en la investigación era voluntaria, que sus respuestas eran anónimas y que podían abandonar el estudio en cualquier momento. Este estudio cumplió con los valores éticos requeridos en la investigación con seres humanos, respetando los principios fundamentales de la Declaración de Helsinki (2008).

\section{Análisis de datos}

Se llevaron a cabo análisis factoriales exploratorios (AFE) y análisis factoriales confirmatorios (AFC), realizando ambos análisis por separado con cada submuestra con la finalidad de disponer de validaciones cruzadas de su estructura factorial (Izquierdo, Olea, \& Abad, 2014; Worthington \& Whittaker, 2006). En primer lugar, se realizó el análisis factorial exploratorio con la primera submuestra utilizando el paquete estadístico SPSS (versión 24, 2016) y se calculó la confiabilidad de los factores. También se exploraron las medias y desviaciones típicas de cada uno de los ítems y su índice de homogeneidad. Previamente a la realización del AFE, se exploró la idoneidad de este análisis mediante la prueba de esfericidad de Barlett y la medida de adecuación de la muestra de Kaiser-Meyer-Olkin (KMO). Para la extracción de factores se utilizó el método de ejes principales con rotación oblimin. Se utilizó la rotación oblimin, puesto que se consideraba probable que existieran correlaciones significativas entre los factores. Para la selección de los factores se tuvo en cuenta que su autovalor fuera superior a 1 y al descenso en el gráfico de sedimentación de Cattell. Como criterio adicional para mantener un factor, se consideró que al menos 3 ítems debían saturar en dicho factor (Hair, Black, Babin, \& Anderson, 2010; Worthington \& Whittaker, 2006).
Posteriormente, se realizó un análisis factorial confirmatorio con la segunda submuestra utilizando el programa MPlus (Muthén \& Muthén, 2010). Para este análisis se utilizó el método de Mínimos Cuadrados Ponderados Robustos (WLSMV) que ofrece el programa MPlus, puesto que es el método más adecuado para trabajar con variables ordinales que se alejen en algún grado de la distribución normal. Por otra parte, teniendo en cuenta las actuales recomendaciones sobre la conveniencia de no utilizar una única medida de ajuste del modelo sino varios índices de diferentes tipos de medidas; hemos considerado, además de Chi-cuadrado (que además puede verse afectado por el tamaño de la muestra) los siguientes índices de ajuste: el índice de ajuste comparativo (CFI), el índice de ajuste no normado de Bentler-Bonett (NNFI) -también llamado índice de Tucker-Lewis (TLI)- y el error de aproximación cuadrático medio (RMSEA). Aunque no deben considerarse como puntos de corte fijos (Bentler, 2007; Markland, 2007), habitualmente son considerados como un buen ajuste del modelo índices de CFI y TLI superiores a .95 y como índices de un ajuste aceptable si superan el .90. En el caso del RMSEA, se considera un buen ajuste a valores inferiores a .50, y un ajuste aceptable valores situados entre .50 y .80 . En cuanto a Chi-cuadrado, se considera que un modelo propuesto ajusta bien a los datos cuando la ratio entre Chi-cuadrado y los grados de libertad es menor a 3 (Bentler \& Bonett, 1980).

Finalmente, para analizar la validez convergente de la escala se realizaron, mediante el cálculo del coeficiente de Pearson, análisis de correlación entre los diferentes factores de la escala de Victimización Escolar entre Iguales y dos variables relacionadas teóricamente con esta variable, como son el autoconcepto social y la satisfacción con la vida. 


\section{Resultados}

\section{Análisis factorial exploratorio}

Los resultados de la prueba de Kaiser-Meyer-Olkin (.91) y de la prueba de esfericidad de Barlett $(\chi 2=4398.24, g l=66, p<.001)$ realizados con la primera submuestra fueron satisfactorios, por lo que se realizó el AFE. Con este análisis se obtuvieron tres factores que explican en conjunto el $58.42 \%$ de la varianza (ver Tabla 1). El primer factor, victimización verbal, incluye ítems relativos principalmente a conductas de agresión verbal sufridas por parte de los compañeros tales como insultos, gritos y burlas. Este factor explica el $43.09 \%$ de la varianza. El segundo factor, victimización física, recoge ítems referidos a agresiones de tipo físico tales como empujones o patadas sufridas en el contexto escolar por parte de los compañeros y explica el $10.26 \%$ de la varianza. $\mathrm{El}$ tercer factor, victimización relacional, incluye ítems que describen situaciones de aislamiento y rechazo social sufridas por parte de los compañeros y explica el 5.07\% de la varianza.

Tabla 1

Medias, DE (desviaciones estándar) e IHc (índices de homogeneidad) de los ítems en la primera submuestra y análisis factorial exploratorio.

\begin{tabular}{|c|c|c|c|c|c|c|}
\hline \multirow[b]{2}{*}{ Algún compañero/a... } & \multirow[t]{2}{*}{ Media } & \multirow[t]{2}{*}{ DE } & \multirow[t]{2}{*}{ IHc } & \multicolumn{3}{|c|}{$\begin{array}{l}\text { Análisis Factorial } \\
\text { Exploratorio }\end{array}$} \\
\hline & & & & $\begin{array}{c}\text { F1 } \\
\text { Violencia } \\
\text { Verbal }\end{array}$ & $\begin{array}{c}\text { F2 } \\
\text { Violencia } \\
\text { Física }\end{array}$ & $\begin{array}{c}\text { F3 } \\
\text { Violencia } \\
\text { Relacional }\end{array}$ \\
\hline $\begin{array}{l}\text { 1. Le ha dicho a los demás que no vayan } \\
\text { conmigo }\end{array}$ & 1.44 & 0.76 & .59 & .10 & -.02 & .63 \\
\hline 2. Me ha apartado de mi grupo de amigos & 1.37 & 0.71 & .64 & -.05 & .09 & .76 \\
\hline $\begin{array}{l}\text { 3. Me ha ignorado o dejado de lado para } \\
\text { que me sienta mal }\end{array}$ & 1.52 & 0.88 & .64 & .01 & .09 & .73 \\
\hline $\begin{array}{l}\text { 4. Ha contado rumores sobre mí y me ha } \\
\text { criticado a mis espaldas }\end{array}$ & 2.21 & 1.22 & .54 & .45 & -.14 & .33 \\
\hline 5. Me ha empujado con fuerza & 1.47 & 0.81 & .63 & .11 & .61 & .04 \\
\hline 6. Me ha pegado o golpeado & 1.33 & 0.72 & .71 & .06 & .75 & .01 \\
\hline 7. Me ha dado una patada & 1.35 & 0.71 & .74 & .01 & .85 & -.04 \\
\hline 8. Me ha tirado al suelo & 1.26 & 0.65 & .65 & -.05 & .72 & .10 \\
\hline 9. Se ha metido conmigo & 1.82 & 1.03 & .76 & .82 & .02 & .03 \\
\hline 10. Me ha insultado & 1.92 & 1.09 & .83 & .94 & .04 & -.07 \\
\hline 11. Se ha burlado de mí & 1.77 & 1.06 & .73 & .68 & .03 & .16 \\
\hline 12. Me ha gritado & 1.73 & 1.07 & .55 & .55 & .16 & -.06 \\
\hline
\end{tabular}


Analizando el contenido de los ítems que se distribuyen en cada uno de los tres factores, se observa una estructura coherente desde el punto de vista teórico, a excepción del ítem 4. Este ítem hace referencia a una agresión indirecta, puesto que las críticas y rumores entre compañeros no se producen de forma directa, sino que se van extendiendo a espaldas de la víctima. Además, observamos también que este ítem muestra cargas factoriales superiores a .32 en dos factores, victimización verbal y victimización relacional. Teniendo cuenta las recomendaciones sobre la eliminación de ítems con saturaciones superiores a .32 en dos o más factores (Worthington \& Whittaker, 2006) y las dificultades de coherencia teórica de este ítem, se decidió eliminarlo de la escala final. Por otra parte, antes de realizar el AFC con la segunda submuestra, se llevó a cabo un nuevo AFE eliminando el ítem 4 para observar la estructura de los datos en esta primera submuestra sin el ítem 4. En este segundo AFE se obtuvieron tres factores que explican en conjunto el $60.12 \%$ de la varianza total: victimización verbal, que explica el $44.35 \%$ de la varianza, victimización física, que explica el $10.18 \%$ de la varianza y victimización relacional, que explica el 5.59\% de la varianza. Los 11 ítems con los que se realizó este segundo AFE saturan en el factor correspondiente desde el punto de vista teórico y su saturación es superior a .32 en un único factor.

Finalmente, se calcularon los coeficientes de consistencia interna (alfa de Cronbach) de los tres factores y se obtuvieron en todos los casos valores superiores a .70. En concreto, se obtuvo un valor de .86 para el factor de victimización verbal (integrado por 4 ítems), .85 para el factor de victimización física (integrado por 4 ítems) y .78 para el factor de victimización relacional (integrado por 3 ítems). Los tres factores muestran entre sí correlaciones positivas ( $r=.55$ entre victimización verbal y victimización física; $r=.57$ entre victimización verbal y victimización relacional; y $r=.40$ entre victimización relacional y victimización física).

\section{Análisis factorial confirmatorio}

A continuación, la estructura factorial de los 11 ítems integrados en estos tres factores fue contrastada mediante un AFC realizado con la segunda submuestra de adolescentes. Los índices de ajuste del modelo propuesto mostraron los siguientes valores: $\chi 2=112.94, g l=41, p<.001$, $\mathrm{CFI}=.991, \mathrm{TLI}=.988$; $\mathrm{RMSEA}=.052(.041-$ .063). Estos valores, tomados en conjunto, indican un buen ajuste del modelo y, por tanto, confirman la estructura factorial de la escala. En la Tabla 2 pueden consultarse las medias, desviaciones típicas e índices de homogeneidad de los 11 ítems de la escala con los datos de esta segunda submuestra, así como también los resultados de la saturación de estos ítems en el factor correspondiente. Los coeficientes de consistencia interna ( $\alpha$ de Cronbach) de estos tres factores en la segunda submuestra fueron también superiores .70, y se obtuvieron valores de .86 para el factor de victimización verbal, .84 para el factor de victimización física y .78 para el factor de victimización relacional. Los tres factores muestran correlaciones positivas $(r=.59$ entre victimización verbal $\mathrm{y}$ victimización relacional; $r=.58$ entre victimización verbal y victimización física y $r=.42$ entre victimización relacional y victimización física). 
Tabla 2

Medias, DE (desviaciones estándar) e IHc (índices de homogeneidad) de los ítems en la segunda submuestra y análisis factorial confirmatorio (solución estandarizada).

\begin{tabular}{|c|c|c|c|c|c|c|}
\hline \multirow[b]{2}{*}{ Algún compañero/a... } & \multirow[t]{2}{*}{ Media } & \multirow[t]{2}{*}{ DE } & \multirow[t]{2}{*}{ IHc } & \multicolumn{3}{|c|}{$\begin{array}{l}\text { Análisis Factorial } \\
\text { Confirmatorio }\end{array}$} \\
\hline & & & & $\begin{array}{c}\text { F1 } \\
\text { Violencia } \\
\text { Verbal }\end{array}$ & $\begin{array}{c}\text { F2 } \\
\text { Violencia } \\
\text { Física }\end{array}$ & $\begin{array}{c}\text { F3 } \\
\text { Violencia } \\
\text { Relacional }\end{array}$ \\
\hline $\begin{array}{l}\text { 1. Le ha dicho a los demás que no vayan } \\
\text { conmigo }\end{array}$ & 1.44 & 0.78 & .59 & & & .76 \\
\hline 2. Me ha apartado de mi grupo de amigos & 1.37 & 0.73 & .64 & & & .84 \\
\hline $\begin{array}{l}\text { 3. Me ha ignorado o dejado de lado para } \\
\text { que me sienta mal }\end{array}$ & 1.53 & 0.89 & .63 & & & .81 \\
\hline 5. Me ha empujado con fuerza & 1.48 & 0.82 & .82 & & .82 & \\
\hline 6. Me ha pegado o golpeado & 1.34 & 0.73 & .73 & & .89 & \\
\hline 7. Me ha dado una patada & 1.36 & 0.73 & .73 & & .88 & \\
\hline 8. Me ha tirado al suelo & 1.27 & 0.67 & .67 & & .83 & \\
\hline 9. Se ha metido conmigo & 1.82 & 1.03 & .76 & .89 & & \\
\hline 10. Me ha insultado & 1.91 & 1.08 & .82 & .93 & & \\
\hline 11. Se ha burlado de mí & 1.77 & 1.06 & .73 & .87 & & \\
\hline 12. Me ha gritado & 1.71 & 1.05 & .51 & .66 & & \\
\hline
\end{tabular}

\section{Análisis de correlación}

Por último, se analizó la validez convergente de la escala considerando las correlaciones entre los tres factores que la componen y otros constructos con los que teóricamente se encuentran relacionados. En concreto, se analizaron las correlaciones de los tres factores de la Escala de Victimización Escolar entre Iguales (VE-I) con la subescala de Autoconcepto Social del AF-5 (García \& Musitu, 1999) y la Escala de Satisfacción con la Vida de Diener et al. (1985). Estos análisis de correlación se realizaron, por separado, con ambas submuestras (ver Tabla 3). Se calcularon además, en ambas submuestras, las correlaciones entre la Subescala de Autoconcepto Social y la Escala de Satisfacción con la Vida. Dada la natu- raleza cuantitativa de las variables consideradas, se optó por la utilización del coeficiente de correlación producto-momento de Pearson. Dicho coeficiente permite evaluar el grado de asociación entre variables. Los valores próximos a 0 indican una ausencia de relación entre variables y la relación entre las variables es cada vez mayor conforme los valores de este coeficiente se aproximan a -1 (correlación negativa) $0+1$ (correlación positiva). Siguiendo las recomendaciones de Cohen (1988) el tamaño del efecto se considera bajo cuando el valor de $r$, aun siendo significativo, se encuentra entre .10 y .30 ; medio con valores situados entre .30 y .50 ; y grande si el valor de $\mathrm{r}$ es superior .50 . 
Tabla 3

Correlaciones entre los factores de la escala de Victimización Escolar entre Iguales (submuestra 1 y submuestra 2), Autoconcepto Social y Satisfacción con la Vida.

\begin{tabular}{|c|c|c|}
\hline & $\begin{array}{c}\text { Autoconcepto } \\
\text { Social }\end{array}$ & $\begin{array}{c}\text { Satisfacción con } \\
\text { la vida }\end{array}$ \\
\hline \multicolumn{3}{|l|}{ Submuestra 1} \\
\hline $\begin{array}{l}\text { Victimización } \\
\text { Verbal }\end{array}$ & -.25 & -.31 \\
\hline $\begin{array}{l}\text { Victimización } \\
\text { Relacional }\end{array}$ & -.28 & -.24 \\
\hline $\begin{array}{l}\text { Victimización } \\
\text { Física }\end{array}$ & -.15 & -.20 \\
\hline $\begin{array}{l}\text { Satisfacción con } \\
\text { la vida }\end{array}$ & .31 & \\
\hline \multicolumn{3}{|l|}{ Submuestra 2} \\
\hline $\begin{array}{l}\text { Victimización } \\
\text { Verbal }\end{array}$ & -.26 & -.30 \\
\hline $\begin{array}{l}\text { Victimización } \\
\text { Relacional }\end{array}$ & -.29 & -.28 \\
\hline $\begin{array}{l}\text { Victimización } \\
\text { Física }\end{array}$ & -.15 & -.25 \\
\hline $\begin{array}{l}\text { Satisfacción con } \\
\text { la vida }\end{array}$ & .30 & \\
\hline
\end{tabular}

Nota. Todas las correlaciones son significativas $(p<.01)$.

En ambas submuestras las correlaciones entre todas las variables consideradas fueron estadísticamente significativas $(p<.01)$ y el signo de estas correlaciones fue el esperado desde un punto de vista teórico. Se observa así cómo en las dos submuestras existen correlaciones negativas significativas entre las tres formas de victimización (física, verbal y relacional) y el autoconcepto social y la satisfacción con la vida. El tamaño del efecto de estas correlaciones es pequeño, a excepción de las relaciones constatadas entre victimización verbal y satisfacción con la vida, cuya correlación es moderada (tamaño del efecto medio) en ambas submuestras. Las correlaciones entre victimización relacional y autoconcepto social, aunque tienen un tamaño del efecto pequeño, se encuentran próximas al punto de corte considerado para correlaciones medias o moderadas $(r$ $=-.28$ primera submuestra; $r=-.29$ segunda submuestra). Por último, se observan también en los adolescentes de ambas submuestras correlaciones significativas positivas y moderadas entre las variables de autoconcepto social y satisfacción con la vida.

\section{Discusión}

El objetivo de este estudio fue analizar las propiedades psicométricas y estructura factorial de la escala de Victimización Escolar entre Iguales (VE-I). Esta escala está basada en la escala previa de Cava et al. (2007), e incluye igualmente ítems relativos a diferentes formas de victimización (física, verbal y relacional), pero cuenta con un menor número de ítems respecto a la escala original. Las graves consecuencias que la victimización entre iguales tiene para el bienestar psicosocial de las víctimas (Guhn et al., 2013; Kerr et al., 2011; Mehari \& Farrell, 2015; Ostrov \& Kamper, 2015; Reijntjes et al., 2010; Thompson \& Leadbeater, 2013; Wu et al., 2015) plantean la necesidad de su investigación $\mathrm{y}$, por tanto, también la necesidad de disponer de instrumentos confiables que permitan su adecuada medición. En este sentido, los resultados de este estudio constatan las adecuadas propiedades psicométricas de la escala de Victimización Escolar entre Iguales (VE-I), y su utilidad para el desarrollo de investigaciones en este ámbito.

Los análisis factoriales realizados en este estudio muestran una estructura compuesta por tres factores. El primer factor, victimización verbal, hace referencia a la victimización que los estudiantes sufren por parte de sus compañeros mediante agresiones de tipo verbal, tales como in- 
sultos, gritos y burlas. El segundo factor, victimización física, evalúa las agresiones de tipo físico, tales como patadas, golpes y empujones, sufridas por los estudiantes y ejercidas por sus compañeros. Finalmente, el tercer factor, victimización relacional, hace referencia a la victimización que los estudiantes sufren mediante situaciones de exclusión social, rechazo y marginación social ejercidas por sus compañeros. Estos tres factores son, además, coincidentes con los factores de la escala de Victimización Entre Iguales de Cava et al. (2007) y con revisiones previas, como las realizadas por Tropp-Gordon (2017) y Wu et al. (2015), en las que se señala que las tres formas principales de victimización entre iguales son la victimización física, la victimización verbal y la victimización relacional. La escala de Victimización Escolar entre Iguales (VE-I) mantiene, por tanto, la misma estructura factorial que la escala de Cava et al. (2007), pero con un menor número de ítems.

La escala de Victimización Escolar entre Iguales (VE-I) muestra, además, adecuados coeficientes de confiabilidad en todos sus factores $y$ correlaciones significativas con el autoconcepto social y con la satisfacción con la vida. Los estudiantes que sufren situaciones de victimización por parte de sus iguales tienen un menor autoconcepto social y muestran menor satisfacción con su vida. Este resultado es coincidente con investigaciones previas en las que se ha relacionado la victimización de los iguales con menor autoconcepto social, más sentimientos de soledad y menor satisfacción vital en los estudiantes (Estévez-López, Martínez-Ferrer, \& Musitu-Ochoa, 2006; Povedano, Cava, Monreal, Varela, \& Musitu, 2015; Proctor, Linley, \& Maltby, 2009; Troop-Gordon, 2017). Los resultados obtenidos señalan, además, correlaciones significativas entre estas dos variables indicativas de bienestar psicosocial (autoconcepto social y satisfacción con la vida) y la victimización de tipo relacional. Este resultado destaca la importancia de incluir este tipo de victimización tanto en la investigación básica como, sobre todo, en las intervenciones dirigidas a la prevención de la victimización entre iguales en contextos escolares.

En este sentido, se constata nuevamente la importancia de disponer de instrumentos confiables para la medición de la victimización entre iguales que tengan en cuenta todas las formas de victimización, sin obviar las consecuencias negativas que para los estudiantes tienen la exclusión y el rechazo social de los compañeros. En los contextos escolares es necesario desarrollar tanto programas de prevención que sensibilicen a estudiantes y profesores contra las diferentes formas de agresión entre compañeros, como también llevar a cabo intervenciones dirigidas a reducir su incidencia y ayudar a los estudiantes que las sufren a afrontar estas situaciones adecuadamente. A este efecto, la escala de Victimización Escolar entre Iguales (VE-I) constituye un instrumento breve y con adecuadas propiedades psicométricas que permite realizar una evaluación confiable de las diferentes formas de victimización entre iguales. La escala VE-I puede, por tanto, ser de utilidad tanto para la investigación sobre variables personales, sociales y escolares relacionadas con la victimización entre iguales como también para detectar situaciones de victimización existentes en el centro y para evaluar la efectividad de las intervenciones realizadas con la finalidad de prevenir y reducir la violencia y victimización entre estudiantes.

El presente estudio cuenta también con algunas limitaciones. En este sentido, aunque se aporta evidencia sobre aspectos importantes relativos a las propiedades psicométricas del instrumento (validez factorial, validez convergente y confiabilidad), otros aspectos psicométricos como el análisis de la confiabilidad test-retest no 
han sido considerados y convendría que lo fueran en futuros estudios. Igualmente, sería conveniente analizar en futuras investigaciones la invarianza factorial de esta escala utilizando para ello muestras de adolescentes más amplias y de diferentes contextos sociales y culturales, y que se consideren muestras de adolescentes de otros países y culturas. No obstante, y a pesar de estas limitaciones, los resultados obtenidos avalan la idoneidad de esta escala para evaluar las diferentes formas de victimización entre iguales en contextos escolares, para analizar las relaciones entre estas diferentes formas de victimización y variables personales, familiares y escolares del alumnado y para evaluar los efectos de intervenciones desarrolladas con el objetivo de prevenir esta problemática.

Ciertamente, en futuras investigaciones sería interesante profundizar en las variables personales, familiares, escolares y sociales relacionadas con la victimización entre iguales, explorar en qué medida el profesorado puede contribuir a reducir la violencia entre estudiantes y analizar las relaciones entre estas formas de victimización, más tradicionales, y la victimización entre iguales a través de las nuevas tecnologías. En relación con esta última cuestión, estudios recientes han vinculado la violencia escolar (física, verbal y relacional) ejercida dentro del centro escolar con la violencia contra compañeros ejercida a través de las redes sociales (Buelga, Martínez-Ferrer, \& Cava, 2017; Kowalski \& Limber, 2013; Ortega-Barón, Buelga, Cava, \& Torralba, 2017). En futuros estudios, deberá prestarse mayor atención a las conexiones existentes entre la victimización más tradicional, realizada en el propio centro escolar, y la victimización entre estudiantes que tiene lugar mediante el uso de las nuevas tecnologías.

Además, es imprescindible desarrollar programas de intervención dirigidos a toda la co- munidad educativa (padres, profesores y estudiantes), con el fin de mejorar su sensibilización ante esta problemática y favorecer su implicación activa ante las diferentes situaciones de violencia entre compañeros. A este efecto, actualmente son numerosos los programas de intervención (Carrascosa, Cava, \& Buelga, 2018; Cerezo, Calvo, \& Sánchez, 2011; Garaigordobil \& Martínez-Valderrey, 2014; Gradinger, Yanagida, Strohmeier, \& Spiel, 2016; Kärnä et al., 2013; Ortega-Baron, 2018; Palladino, Nocentini, \& Menesini, 2016) que tienen en cuenta la implicación del profesorado, que buscan sensibilizar ante las consecuencias de las diferentes formas de violencia y que tratan de potenciar en el alumnado aquellos recursos personales y sociales (empatía, autoestima, habilidades sociales y resolución positiva de conflictos, entre otros) que favorecen la integración social, el afrontamiento efectivo del acoso y la movilización de los espectadores para ayudar a la víctima.

La implementación en los centros escolares de este tipo de programas de intervención resulta necesaria, puesto que la victimización entre iguales presenta una alta estabilidad temporal y algunos estudiantes sufren de forma continuada y durante varios cursos académicos las agresiones físicas, verbales y relacionales de sus compañeros (Pouwels et al., 2016). No obstante, es también fundamental hacer una evaluación rigurosa de la efectividad de las intervenciones realizadas en los diferentes centros educativos. Los programas y estrategias de prevención implementados deben contar con una evaluación rigurosa de su efectividad mediante la utilización de instrumentos confiables. En este sentido, los resultados de este estudio muestran que la escala VE-I puede ser un instrumento adecuado para evaluar la efectividad de estas intervenciones, al realizar una evaluación confiable de las diferentes formas de victimización escolar entre iguales y permitir una valora- 
ción de los cambios conseguidos a través de la intervención.

\section{Referencias}

Atienza, F. L., Pons, D., Balaguer, I., \& García-Merita, M. (2000). Propiedades psicométricas de la Escala de Satisfacción con la Vida en adolescentes. Psicothema, 12(2), 314-320. Recuperado de http://www. psicothema.com

Bentler, P. M. (2007). On tests and indices for evaluating structural models. Personality and Individual Differences, 42(5), 825-829. doi: 10.1016/j. paid.2006.09.024

Bentler, P. M., \& Bonett, D. G. (1980). Significance tests and goodness of fit in the analysis of covariance structures. Psychological Bulletin, 88(3), 588-606. doi: 10.1037/0033-2909.88.3.588

Bradshaw, J., Crous, G., Rees, G., \& Turner, N. (2017). Comparing children's experiences of school-based bullying across countries. Children and Youth Services Review, 80, 171-180. doi: 10.1016/j.childyouth. 2017.06 .060

Buelga, S., Cava, M. J., \& Musitu, G. (2012). Reputación social, ajuste psicosocial y victimización entre adolescentes en el contexto escolar. Anales de Psicología, 28(1), 180-187. Recuperado de http://revistas. um.es/analesps/index

Buelga, S., Martínez-Ferrer, B., \& Cava, M. J. (2017). Differences in family climate and family communication among cyberbullies, cybervictims, and cyber bully-victims in adolescents. Computers in Human Behavior, 76, 164-173. doi: 10.1016/j. chb.2017.07.017

Card, N. A., \& Hodges, E. V. E. (2008). Peer victimization among schoolchildren: Correlations, causes, consequences, and considerations in assessment and intervention. School Psychology Quarterly, 23(4), 451461. doi: 10.1037/a0012769

Carrascosa, L., Cava, M. J., \& Buelga, S. (2018). Programa
DARSI: Desarrollando en Adolescentes Relaciones Saludables e Igualitarias. Valencia: Palmero.

Cava, M. J. (2011). Familia, profesorado e iguales: Claves para el apoyo a las víctimas de acoso escolar. Psychosocial Intervention, 20(2), 183-192. doi: 10.5093/ in $2011 \mathrm{v} 20 \mathrm{n} 2 \mathrm{a} 6$

Cava, M. J., Buelga, S., Musitu, G., \& Murgui, S. (2010). Violencia escolar entre adolescentes y sus implicaciones en el ajuste psicosocial: Un estudio longitudinal. Revista de Psicodidáctica, 15(1), 21-34. Recuperado de http://www.ehu.eus/ojs/index.php/ psicodidactica/index

Cava, M. J., Musitu, G., Buelga, S., \& Murgui, S. (2010). The relationships of family and classroom environments with peer relational victimization: An analysis of their gender differences. The Spanish Journal of Psychology, 13(1), 156-165. doi: 10.1017/ S1138741600003747

Cava, M. J., Musitu, G., \& Murgui, S. (2007). Individual and social risk factors related to victimization in a sample of Spanish adolescents. Psychological Reports, 101(1), 275-290. doi: 10.2466/pr0.101.1.275290

Cerezo, F., Calvo, A. R., \& Sánchez, C. (2011). Programa CIP. Intervención psicoeducativa y tratamiento diferenciado del bullying. Concienciar, Informar y Prevenir. Madrid, España: Pirámide.

Cohen, J. (1988). Statistical power analysis for the behavioral sciences ( $2^{\mathrm{a}}$ ed.). Hillsdale, NJ, Estados Unidos: Lawrence Erlbaum Associates.

Crick, N. R., \& Grotpeter, J. K. (1996). Children's treatment by peers: Victims of relational and overt aggression. Development and Psychopathology, 8(2), 367-380. doi: 10.1017/S0954579400007148

Declaración de Helsinki (2008). Principios éticos para las investigaciones médicas en seres humanos. Declaración aprobada en la $59^{\mathrm{a}}$ Asamblea General de la Asociación, celebrada en Seúl, Corea. http://bvs.sld. cu/revistas/recursos/helsinki.pdf

Del Barrio, C., Martín, E., Montero, I., Gutiérrez, H., Barrios, A., \& De Dios, M. J. (2008). Bullying and so- 
cial exclusion in Spanish secondary schools: National trends from 1999 to 2006. International Journal of Clinical and Health Psychology, 8(3), 657-677. Recuperado de http://www.aepc.es/ijchp/indizacion. php? coid $=$ Espa $\%$ EF $\%$ BF $\%$ BDol

Del Rey, R., \& Ortega, R. (2008). Bullying in poor countries: Prevalence and coexistence with other forms of violence. International Journal of Psychology and Psychological Therapy, 8(1), 39-50. Recuperado de http://www.ijpsy.com/

Diener, E., Emmons, R., Larsen, R. J., \& Griffin, S. (1985). The Satisfaction with Life Scale. Journal of Personality Assessment, 49(1), 71-75. doi: 10.1207/ s15327752jpa4901_13

Estévez, E., Jiménez, T. I., \& Cava, M. J. (2016). A cross-cultural study in Spain and Mexico on school aggression in adolescence: Examining the role of individual, family and school variables. Cross-Cultural Research, 50(2), 123-153. doi: 10.1177/1069397115625637

Estévez, E., Jiménez, T. I., \& Moreno, D. (2010). Cuando las víctimas de violencia escolar se convierten en agresores: “¿Quién va a defenderme?”. European Journal of Education and Psychology, 3(2), 177-186. doi: 10.30552/ejep.v3i2.40

Estévez-López, E., Martínez-Ferrer, B., \& Musitu-Ochoa, G. (2006). La autoestima en adolescentes agresores y víctimas en la escuela: La perspectiva multidimensional. Psychosocial Intervention, 15(2), 223-232. Recuperado de http://scielo.isciii.es/scielo.php?script $=$ sci_serial\&pid=1132-0559\&lng=es\&nrm=iso

Garaigordobil, M., \& Martínez-Valderrey, V. (2014). Efecto del Cyberprogram 2.0 sobre la reducción de la victimización y la mejora de la competencia social en la adolescencia. Revista de Psicodidáctica, 19(2), 289305. doi: 10.1387/RevPsicodidact.10239

García, F., \& Musitu, G. (1999). AF5: Autoconcepto Forma 5. Madrid, España: TEA Ediciones.

Gradinger, P., Yanagida, T., Strohmeier, D., \& Spiel, C. (2016). Effectiveness and sustainability of the ViSC Social Competence Program to prevent cyberbullying and cyber-victimization: Class and individual level moderators. Aggressive Behavior, 42(2), 181193. doi: 10.1002/ab.21631

Guhn, M., Schonert-Reichl, K. A., Gadermann, A. M., Hymel, S., \& Hertzman, C. (2013). A population study of victimization, relationships, and well-being in middle childhood. Journal of Happiness Studies, 14(5), 1529-1541. doi: 10.1007/s10902-012-9393-8

Hair, J. F. Jr., Black, W. C., Babin, B. J., \& Anderson, R. E. (2010). Multivariate data analysis ( $7^{\mathrm{a}} \mathrm{ed}$.). Upper Saddle River, NJ, Estados Unidos: Prentice Hall.

IBM Corp. (2016). IBM SPSS Statistics for Windows, Version 24.0 [software de cómputo]. Armonk, NY: IBM Corp.

Izquierdo, I., Olea, J., \& Abad, F. J. (2014). Exploratory factor analysis in validation studies: Uses and recommendations. Psicothema, 26(3), 395-400. Recuperado de http://www.psicothema.com

Jiménez, T. I., Musitu, G., Ramos, M. J., \& Murgui, S. (2009). Community involvement and victimization at school: An analysis through family, personal and social adjustment. The Journal of Community Psychology, 37(8), 959-974. doi: 10.1002/jcop.20342

Kärnä, A., Voeten, M., Little, T. D., Alanen, E., Poskiparta, E., \& Salmivalli, C. (2013). Effectiveness of the KiVa antibullying program: Grades 1-3 and 7-9. Journal of Educational Psychology, 105(2), 535551. doi: 10.1037/a0030417

Kerr, J. C., Valois, R. F., Huebner, E. S., \& Drane, J. W. (2011). Life satisfaction and peer victimization among USA public high school adolescents. Child Indicators Research, 4(1), 127-144. doi: 10.1007/ s12187-010-9078-y

Kljakovic, M., \& Hunt, C. (2016). A meta-analysis of predictors of bullying and victimisation in adolescence. Journal of Adolescence, 49, 134-145. doi: 10.1016/j. adolescence.2016.03.002

Kowalski, R. M., \& Limber, S. P. (2013). Psychological, physical, and academic correlates of cyberbullying and traditional bullying. Journal of Adolescent Health, 53(1), S13-S20. doi: 10.1016/j.jadohealth.2012.09.018 
Markland, D. (2007). The golden rule in that there are no golden rules: A commentary on Paul Barrett's recommendations for reporting model fit in structural equation modelling. Personality and Individual Differences, 42(5), 851-858. doi: 10.1016/j.paid.2006.09.023

Mehari, K. R., \& Farrell, A. D. (2015). The relation between peer victimization and adolescents' well-being: The moderating role of ethnicity within context. Journal of Research on Adolescence, 25(1), 118-134. doi: 10.1111/jora.12095

Muthén, B. O., \& Muthén, L. K. (2010). Mplus User's Guide (6 $6^{\mathrm{a}}$ ed.). Los Ángeles, CA, Estados Unidos: Muthén \& Muthén.

Mynard, H., \& Joseph, S. (2000). Development of the multidimensional peer-victimization scale. Aggressive Behavior, 26(2), 169-178. doi: $\quad 10.1002 /(\mathrm{SICI}) 1098-2337(2000) 26: 2 \%$ 3C169::AID-AB3\%3E3.0.CO;2-A

Ortega-Baron, J. (2018). Prevención del acoso en adolescentes a través de las nuevas tecnologías de la información y de la comunicación: ProgramaPreva@cib (Tesis Doctoral no publicada). Universidad de Valencia, España. Resumen disponible en https://dialnet. unirioja.es/servlet/tesis? codigo $=137828$

Ortega-Barón, J., Buelga, S., Cava, M. J., \& Torralba, E. (2017). Violencia escolar y actitud hacia la autoridad de estudiantes agresores de cyberbullying. Revista de Psicodidáctica, 22(1), 23-28.

Ostrov, J. M., \& Kamper, K. E. (2015). Future directions for research on the development of relational and physical peer victimization. Journal of Clinical Child \& Adolescent Psychology, 44(3), 509-519. doi: 10.1080/15374416.2015.1012723

Palladino, B. E., Nocentini, A., \& Menesini, E. (2016). Evidence-based intervention against bullying and cyberbullying: Evaluation of the NoTrap! program in two independent trials. Aggressive Behavior, 42(2), 194-206. doi: 10.1002/ab.21636

Pouwels, J. L., Souren, P. M., Lansu, T. A. M., \& Cillessen A. H. N. (2016). Stability of peer victimization: A meta-analysis of longitudinal research. Developmen- tal Review, 40, 1-24. doi: 10.1016/j.dr.2016.01.001

Povedano, A., Cava, M. J., Monreal, M. C., Varela, R., \& Musitu, G. (2015). Victimization, loneliness, overt and relational violence at the school from a gender perspective. International Journal of Clinical and Health Psychology, 15(1), 44-51. doi: 10.1016/j.ijchp.2014.09.001

Proctor, C. L., Linley, P. A., \& Maltby, J. (2009). Youth life satisfaction: A review of the literature. Journal of Happiness Studies, 10(5), 583-630. doi: 10.1007/ s10902-008-9110-9

Reijntjes, A., Kamphuis, J. H., Prinzie, P., \& Telch, M. J. (2010). Peer victimization and internalizing problems in children: A meta-analysis of longitudinal studies. Child Abuse \& Neglect, 34(4), 244-252. doi: 10.1016/j.chiabus.2009.07.009

Thompson, R. S. Y., \& Leadbeater, B. J. (2013). Peer victimization and internalizing symptoms from adolescence into young adulthood: Building strength through emotional support. Journal of Research on Adolescence, 23(2), 290-303. doi: 10.1111/j.15327795.2012.00827.x

Troop-Gordon, W. (2017). Peer victimization in adolescence: The nature, progression, and consequences of being bullied within a developmental context. Journal of Adolescence, 55, 116-128. doi: 10.1016/j.adolescence.2016.12.012

Worthington, R. L., \& Whittaker, T. A. (2006). Scale development research. A content analysis and recommendations for best practices. The Counseling Psychologist, 34(6), 806-838. doi: 10.1177/0011000006288127

Wu, L., Zhang, D., Su, Z., \& Hu, T. (2015). Peer victimization among children and adolescents: A meta-analytic review of links to emotional maladjustment. Clinical Pediatrics, 54(10), 941-955. doi: 10.1177/0009922814567873 